Clinical Research

\title{
High expression of Sterol-O-Acyl transferase 1 (SOAT1), an enzyme involved in cholesterol metabolism, is associated with earlier biochemical recurrence in high risk prostate cancer
}

\author{
Carolin Eckhardt ${ }^{1}$, Iuliu Sbiera ${ }^{1}$, Markus Krebs ${ }^{2,3}$, Silviu Sbiera (D) ${ }^{1}$, Martin Spahn ${ }^{4,5}$, Burkhard Kneitz ${ }^{2}$, Steven Joniau (iD ${ }^{6}$, \\ Martin Fassnacht ${ }^{1,3}$, Hubert Kübler ${ }^{2,3}$, Isabel Weigand ${ }^{1,7}$ and Matthias Kroiss (DD ${ }^{1,3,7}$ \\ (c) The Author(s) 2021
}

BACKGROUND: Prostate cancer (PCa) is the most frequent cancer in men. The prognosis of PCa is heterogeneous with many clinically indolent tumors and rare highly aggressive cases. Reliable tissue markers of prognosis are lacking. Active cholesteryl ester synthesis has been associated with prostate cancer aggressiveness. Sterol-O-Acyl transferases (SOAT) 1 and 2 catalyze cholesterol esterification in humans.

OBJECTIVE: To investigate the value of SOAT1 and SOAT2 tissue expression as prognostic markers in high risk PCa.

PATIENTS AND METHODS: Formalin-fixed paraffin-embedded tissue samples from 305 high risk PCa cases treated with radical prostatectomy were analyzed for SOAT1 and SOAT2 protein expression by semi-quantitative immunohistochemistry. The Kaplan-Meier method and Cox proportional hazards modeling were used to compare outcome.

MAIN OUTCOME MEASURE: Biochemical recurrence (BCR) free survival.

RESULTS: SOAT1 expression was high in 73 (25\%) and low in 219 (75\%; not evaluable: 13) tumors. SOAT2 was highly expressed in $40(14 \%)$ and at low levels in 249 (86\%) samples (not evaluable: 16). By Kaplan-Meier analysis, we found significantly shorter median BCR free survival of 93 months (95\% confidence interval 23.6-123.1) in patients with high SOAT1 vs. 134 months $(112.6-220.2$, Log-rank $p<0.001)$ with low SOAT1. SOAT2 expression was not significantly associated with BCR. After adjustment for age, preoperative PSA, tumor stage, Gleason score, resection status, lymph node involvement and year of surgery, high SOAT1 but not SOAT2 expression was associated with shorter BCR free survival with a hazard ratio of $2.40(95 \% \mathrm{Cl} 1.57-3.68, p<0.001)$. Time to clinical recurrence and overall survival were not significantly associated with SOAT1 and SOAT2 expression

CONCLUSIONS: SOAT1 expression is strongly associated with BCR free survival alone and after multivariable adjustment in high risk PCa. SOAT1 may serve as a histologic marker of prognosis and holds promise as a future treatment target.

Prostate Cancer and Prostatic Diseases (2022) 25:484-490; https://doi.org/10.1038/s41391-021-00431-3

\section{INTRODUCTION}

Prostate cancer (PCa) is the most frequent cancer in men [1]. Apart from its high incidence, PCa heterogeneity poses a major challenge in clinical care. While the incidence of PCa has increased in the western world as a result of widespread screening programs using prostate specific antigen (PSA) as a serum marker [2], mortality rates did not decrease substantially [3]. This reflects that the majority of PCa patients has indolent (low risk) cancers while the small subgroup of lethal (high risk) cancers cannot be reliably identified and treated after positive PSA screening. Prognostic markers are hence of major relevance. The current strategy to identify high risk prostate cancer (high risk PCa) cases uses a combination of clinical and histological markers because it has been shown that PCa-related death can be predicted by using tumor stage $3 b-4$, Gleason score $\geq 8$ and a positive lymph node status as markers of unfavorable prognosis [4].

Accumulating data suggest that features of tumoral metabolism may be prognostically relevant and be of therapeutic relevance [5]. By using RAMAN-spectroscopy of high risk PCa samples, accumulation of cholesteryl esters (CE) has been shown to be a hallmark of PCa progression. Experimental data suggest that loss of the tumor suppressor PTEN, a known driver of PCa, might be causally related to increased cholesterol metabolism [6]. Inhibition of cholesteryl ester formation with avasimibe, an inhibitor of Sterol-O-Acyl transferase 1 (SOAT1, also known as Acyl-CoACholesterol Acyl transferase 1, ACAT1) in a cell culture model of PCa has been shown to reduce cell viability and in vitro indicators of cell migration and invasiveness. Subsequent research in a

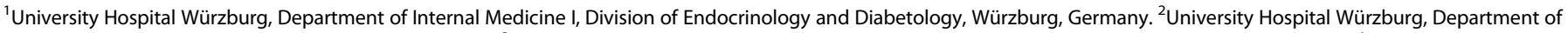

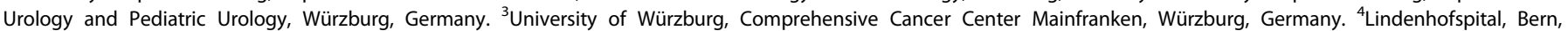

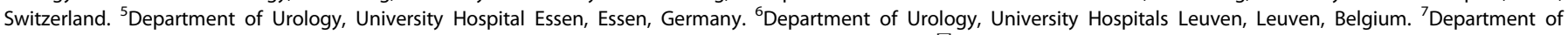
Medicine IV, University Hospital Munich, Ludwig-Maximilians-Universität München, München, Germany. ${ }^{\bowtie}$ email: Kroiss_M@ukw.de 
mouse model of metastatic PCa treated with injectable avasimibe showed that depletion of CE resulted in abrogated Wnt signaling [7] through an indirect mechanism involving suppression of fatty acid synthesis and decreased Wnt3 acylation. While the relationship of cholesterol metabolism and tumoral androgen synthesis in PCa is still controversial $[8,9]$, available data suggest that targeting CE formation may be a promising approach for treatment of aggressive PCa $[6,7]$.

Intracellular synthesis of $C E$ in humans from cholesterol and Acyl-CoA is catalyzed by SOAT1 and SOAT2. According to current understanding, SOAT1 and 2 exhibit functional differences: SOAT1 is expressed at variable levels in many tissues and preferentially catalyzes the esterification of free cholesterol with unsaturated fatty acids [10] that are stored as intracellular lipid droplets (LD). LDs hence serve as buffers for free cholesterol, which would otherwise impair cellular function by interfering with membrane fluidity [11]. SOAT2 is mainly expressed in liver and intestine and plays a role in secretion of lipoproteins $[12,13]$.

We have previously shown that SOAT1 inhibition is the main molecular mechanism of mitotane, the only drug approved for treatment of adrenocortical carcinoma [14]. The selective SOAT1 inhibitor nevanimibe (ATR-101, PD132301-2) has been investigated for the treatment of adrenal disorders $[15,16]$ and was recently tested in a phase I clinical trial against adrenocortical carcinoma [17].

Protein expression of SOAT1 and SOAT2 has not been examined in prostate cancer tissue. In the present study, we investigated the expression of both enzymes in a large cohort of well characterized high risk PCa tissue samples from two centers and demonstrate strong association of SOAT1 expression with poor prognosis. We used time to biochemical recurrence (BCR), a strong predictor of cancerspecific mortality in high risk $\mathrm{PCa}$, as the primary end point [18].

\section{PATIENTS AND METHODS \\ Patients and tissue}

305 formalin-fixed-paraffin-embedded (FFPE) high risk PCa samples (defined as PSA $>20 \mathrm{ng} / \mathrm{mL}$ and/or clinical stage T3/4 and/or biopsy Gleason score 8-10) who had undergone radical prostatectomy between 1987 and 2005 at two clinical centers (Karlsruhe, Germany: 206 samples, Leuven, Belgium: 99 samples) were included in this study. Detailed clinical data were collected in the European Clinical and Translational High-Risk Prostate Cancer Research Group database (EMPaCT) as described before [19]. All patients underwent preoperative staging with digital rectal examination (DRE), abdominopelvic computed tomography scan and bone scan. PCa was confirmed by transrectal biopsy and pretreatment PSA was measured before DRE. None of the patients had received neo-adjuvant radiation or chemotherapy. After surgery, the absence or presence of local recurrence or distant metastasis (clinical progression) was evaluated every three months for the first two years, every six months in the following three years and annually after by computerized tomography or bone-scan. $B C R$ was defined by prostate-specific antigen (PSA) of greater than $0.2 \mathrm{ng} /$ $\mathrm{ml}$ in two consecutive measurements. Overall survival (OS) was defined as time from radical prostatectomy to death of any cause or last follow-up visit, while cancer-specific survival (CSS) was defined as death caused by PCa or complications of PCa. With a known overall BCR rate of $\sim 35 \%$ in our available high risk PCa cohort, 116 events would be necessary to observe a clinically relevant difference in the incidence of $25 \%$ vs. $50 \%$ BCR with a power of $80 \%$ and an alpha error of 0.05 .

Written informed consent was obtained from all patients and the study was approved by the cantonal ethics committee in Bern (128/2015; 26.05.2015) and performed in accordance with the declaration of Helsinki. Median follow-up was 89 months (range 6-200).

Bioinformatical analysis of the TCGA (The Cancer Genome Atlas) Research Network, specifically its primary PCa (PRAD) cohort, and the DreamTeam cohort [20] consisting of PCa metastases was carried out with data retrieved from the cBioPortal [21].

\section{Immunohistochemistry}

Tissue samples were assembled into five tissue microarrays (TMA) and up to four cores were available per sample and punch diameter was $0.6 \mathrm{~mm}$.
Slides of $4 \mu \mathrm{m}$ were deparaffinized twice in $100 \%$ xylene (Sigma-Aldrich, Taufkirchen, Germany) for $12 \mathrm{~min}$ and rehydrated in a descending ethanolseries, followed by washing with distilled water. Antigen retrieval was performed in $10 \mathrm{mM}$ citric acid monohydrate buffer $(\mathrm{pH} 6.5)$ for $13 \mathrm{~min}$ in a pressure cooker. Quenching of endogenous peroxidase activity was done with $3 \% \mathrm{H}_{2} \mathrm{O}_{2}$ for 10 min. Unspecific binding sites were blocked with $20 \%$ human $A B$ serum for $1 \mathrm{~h}$ at room temperature. Subsequently, SOAT enzymes were detected by incubation with polyclonal rabbit primary antibodies (SOAT1-antibody, abcam, Cambridge, UK, ab39327, 1:1000 in PBS over night at $4{ }^{\circ} \mathrm{C}$; SOAT2-antibody, Acris Antibodies $\mathrm{GmbH}$, Herford, Germany, Cayman Chemicals 100027, 1:400 in PBS for $1 \mathrm{~h}$ at room temperature). Signal amplification was achieved by using Advance-HRP-Kit (Dako, Hamburg, Germany) and development for 10 min with Liquid DAB + Substrate Chromogen System (K3468, Dako) according to the manufacturer's instructions. Samples were counterstained with Mayer's hemalaun for two minutes followed by washing in tap water.

\section{Microscopy and quantification of expression}

All slides were analyzed independently by two investigators (C.E. and I.S.) by using semi-quantitative $\mathrm{H}$-scoring as described [22]. Discrepancies in scoring were double checked by both investigators and a consensus reached.

Only cytoplasmic staining of tumor cells was considered and staining intensity graded as negative (0), low (1), medium (2), and strong (3). A proportion score was calculated by determining the percentage of positive tumor cells for each sample which was 0 if $0 \%$ tumor cells were positive, 0.1 if $1-9 \%$ were positive, 0.5 if $10-49 \%$ were positive and 1 if $\geq 50 \%$ were positive. $\mathrm{H}$-score was then calculated by multiplying the staining intensity grading score with the proportion score (range $0-3$ ) taking into account all TMA cores. $\mathrm{H}$-score of $<3$ vs. 3 was considered as low and high SOAT expression, respectively.

\section{Statistics and bioinformatical analyses}

The interobserver agreement was evaluated using Cohen's K. The Correlation between SOAT H-Score and histological Gleason score was analyzed using Spearman's rank correlation test. Survival was estimated using the Kaplan-Meier method and groups were compared by log rank test. Cox proportional hazards modeling included age, tumor stage, preoperative PSA, Gleason score, resection status, lymph node status and year of surgery. The significance level was set as $p<0.05$ for all comparisons. All statistical tests were performed using SPSS 26.0 (IBM, Armonk, NY) or Prism 6 (GraphPad, San Diego, CA). Co-expression analyses of SOAT1 and genes related to androgen signaling [23]. and cholesterol biosynthesis [24] were performed via cbioportal [21, 25]-Spearman's rank correlation coefficients were considered significant after performing BenjaminiHochberg correction.

\section{RESULTS}

\section{Patient characteristics}

The study cohort is described in Tables 1 and S1. Median age at surgery was 66 years (range 41-81) and median histopathological Gleason score 7 (range 3-9). After prostatectomy, resection was considered complete (R0) in 123 patients (40.3\%), R1 in 147 and unknown (RX) in 35 patients (11.5\%). Median follow-up was 89 months (range 6-200) after radical prostatectomy. 109 patients experienced biochemical recurrence after a median of 26 months (range 1-166), 44 had clinical progression after a median of 36 months (range 3-144) and 22 died of PCa (57 months, 14-166).

\section{Immunohistochemical detection of SOAT}

Both SOAT1 and SOAT2 staining was localized to the cytoplasmic region of prostate cancer cells consistent with their known localization at the endoplasmic reticulum. Figure 1 shows examples of negative ( $\mathrm{H}$-score 0$)$, low (H-score 1$)$, intermediate (H-score 2 ) and high ( $\mathrm{H}$-score 3 ) expression of SOAT1 (Fig. $1 \mathrm{~A}-\mathrm{H}$ ) and SOAT2 (Fig. 1I-P). Both SOAT1 and SOAT2 were homogeneously distributed among the different cores in the TMA. Interrater reliability for assessing SOAT expression was strong and very strong with a k-coefficient of $0.66(95 \% \mathrm{Cl} 0.61-0.70)$ for SOAT1 and 0.91 (95\% Cl 0.89-0.94) for SOAT2, respectively. 
Table 1. Clinico-pathological features of the study population.

\begin{tabular}{|c|c|}
\hline Variable & Median/number \\
\hline Age at surgery (range) & $66(41-81)$ \\
\hline Pre-operative PSA, ng/ml (range) & $30(1-597)$ \\
\hline \multicolumn{2}{|l|}{ Clinical stage } \\
\hline $\mathrm{T} 2$ & $59(19.3 \%)$ \\
\hline T3 & $203(66.6 \%)$ \\
\hline T4 & $43(14.1 \%)$ \\
\hline \multicolumn{2}{|l|}{ Gleason-score } \\
\hline$\leq 7$ & $222(77.6 \%)$ \\
\hline $8-10$ & $64(22.4 \%)$ \\
\hline \multicolumn{2}{|l|}{ Resection margin } \\
\hline Ro & $123(40.3 \%)$ \\
\hline R1 & $147(48.2 \%)$ \\
\hline RX & $35(11.5 \%)$ \\
\hline \multicolumn{2}{|l|}{ Lymph node status } \\
\hline NO & $211(69.4 \%)$ \\
\hline N1 & $93(30.6 \%)$ \\
\hline \multicolumn{2}{|l|}{ SOAT1 } \\
\hline \multicolumn{2}{|l|}{ expression score } \\
\hline$<3$ & $219(75.0 \%)$ \\
\hline 3 & $73(25.0 \%)$ \\
\hline \multicolumn{2}{|l|}{ SOAT2 } \\
\hline \multicolumn{2}{|l|}{ expression score } \\
\hline$<3$ & $249(86.2 \%)$ \\
\hline 3 & $40(13.8 \%)$ \\
\hline \multicolumn{2}{|l|}{ BCR } \\
\hline In total & $109(35.7 \%)$ \\
\hline Median time to $\mathbf{B C R}$, month (range) & $25.5(1-166)$ \\
\hline \multicolumn{2}{|l|}{ Clinical progression } \\
\hline In total & $44(14.4 \%)$ \\
\hline $\begin{array}{l}\text { Median time to clinical progression, month } \\
\text { (range) }\end{array}$ & $35.5(3-144)$ \\
\hline Death for any reason & $30(10.0 \%)$ \\
\hline Cancer related death & $22(7.2 \%)$ \\
\hline
\end{tabular}

$n=305$; only data included with complete clinical data and evaluable tissu. PSA prostate-specific antigen, SOAT Sterol-O-Acyl transferase, BCR biochemical recurrence.

Overall, $96 \%$ of SOAT1 and $95 \%$ of SOAT2 stained TMA cores could be assessed and SOAT1 and SOAT2 expression was detectable in more than $99 \%$ each. $25 \%$ of the samples showed strong $(n=73, \mathrm{H}$-score 3$)$ and $75 \%$ weak $(n=219, \mathrm{H}$-score $<3)$ SOAT1 expression. For SOAT2, $14 \%$ of the cores showed high $(n=$ $40, \mathrm{H}$-score 3$)$ and $86 \%$ low $(n=249, \mathrm{H}$-score $<3)$ expression. SOAT1 and SOAT2 protein expression were moderately correlated (Spearman Rho $=0.339, p<0.001)$.

\section{Impact of SOAT protein expression on BCR free survival}

For survival analysis, only patients with complete clinical data and evaluable tissue were included. BCR was observed in 39 of 73 (53.4\%) samples with high SOAT1 expression and $63(28.7 \%)$ samples with low SOAT1 expression. 19 of 40 (47.5\%) samples with high SOAT2 expression and 83 (33.3\%) samples with low SOAT2 expression had BCR during a median follow-up of 89 months.

Kaplan-Meier analysis revealed a significantly shorter median BCR free survival of 93 months (95\% confidence interval $[\mathrm{CI}] 23.6-$
123.1 months) in patients with high SOAT1 ( $n=72$, Fig. 2A) compared to 134 (95\% Cl 112.6-220.2 months) in patients with low SOAT1 expression $(n=218$, Log-rank $p<0.001)$.

Patients with high tumoral SOAT2 expression (H-score $3, n=40$, Fig. 2B) showed a trend for shorter BCR with a median of 91 months $(95 \% \mathrm{Cl}$ not computed) compared to 125 (111.5-158.5 months) in patients with low SOAT2 staining $(n=$ 247, Log rank $p=0.05$ ).

To account for known prognostic factors in PCa including year of surgery, we performed Cox proportional hazards modeling with SOAT1 and age at surgery, pre-operative PSA, tumor stage, Gleason score, surgical margin status, lymph node status and year of surgery (Table 2). High SOAT1 expression retained significant association with less favorable BCR after multivariable adjustment (HR 2.40, 95\% Cl 1.57-3.68, $p<0.001$ ). Earlier BCR in patients with Gleason score $\geq 8$ approached statistical significance with a hazard ratio of $1.57(95 \% \mathrm{Cl} 0.99-2.49$, Log-rank $p=0.06)$. SOAT2 expression was not significantly associated with BCR (HR 1.57, $95 \% \mathrm{Cl} 0.93-2.64$, Log-rank $p=0.09$ ). SOAT staining and Gleason score were not significantly correlated (Pearson $r=0.042, p=0.49$ ).

\section{Impact of SOAT1 and SOAT2 expression on secondary endpoints}

Clinical recurrence-free survival was not significantly associated with high vs. low SOAT1 expression (median 167 months for both groups, Log-rank $p=0.816$ ) nor was overall survival (high SOAT 1:172 months, low SOAT1: 174 months, Log-rank $P=0.476$ ).

High vs. low SOAT2 expression was not significantly associated with clinical recurrence-free (162 vs. 171 months, Log-rank $p=0.346$ ) and overall survival (177 vs. 171 months, Log-rank $p=0.962$ ).

\section{Correlation of SOAT1 with lipid metabolism and androgen receptor (AR) signaling}

In the TCGA and DreamTeam [20] cohorts, squalene epoxidase (SQLE) had a moderate significant positive correlation with SOAT1 (Spearman $\rho=0.20, p<0.001$ and $p=0.21, p<0.01$; Fig. S1A, B). In line with an increased lipid metabolism, low density lipoprotein receptor (LDLR) and SOAT1 were also moderately positively correlated (Spearman $p=0.38, p<0.001$ and $\rho=0.26, p<0.001$ ) in the TCGA and DreamTeam cohorts, respectively (Fig. S1C, D) and so were stearoyl-CoA desaturase (SCD) and SOAT1 (Spearman $\rho=0.25, P<0.001$ and $\rho=0.33, p<0.001$; Fig. S1E, F and Table S2). To explore the interconnection between SOAT1 expression and androgen receptor (AR) signaling on $\mathrm{PCa}$ progression [26] we examined the gene expression of SOAT1 and $A R$ and found a good and statistically significant correlation in both the TCGA (Spearman $\rho=0.53, p<0.001$, Fig. S2A) and the DreamTeam cohort (Spearman $\rho=0.3, p<0.01$, Fig S2B). Accordingly, several AR signaling related genes [23] were consistently correlated with SOAT1 expression in both cohorts (Table S3).

\section{DISCUSSION}

$\mathrm{PCa}$ is the most frequent cancer in men but the overall mortality risk is low with only approximately $3 \%$ in western countries. However, about 15 to $30 \%$ of patients present with high risk PCa which is associated with distinctly higher aggressiveness and mortality rates. In addition, clinical outcome and likelihood of BCR vary due to different classification systems of high risk PCa [27-34]. Beyond established prognostic and clinical parameters such as Gleason score, PSA or tumor stage, reliable biomarkers for the prognostication of high risk PCa are still lacking, especially those further explaining high risk PCa biology. Yet, such prognostic and functional markers are crucial first to identify patients that are more likely to experience relapse and second to better stratify patients for personalized treatment approaches [19, 35]. Here, we evaluated the association of tissue protein expression of SOAT1 and SOAT2 in 305 high risk PCa patients on clinical parameters of recurrence. 

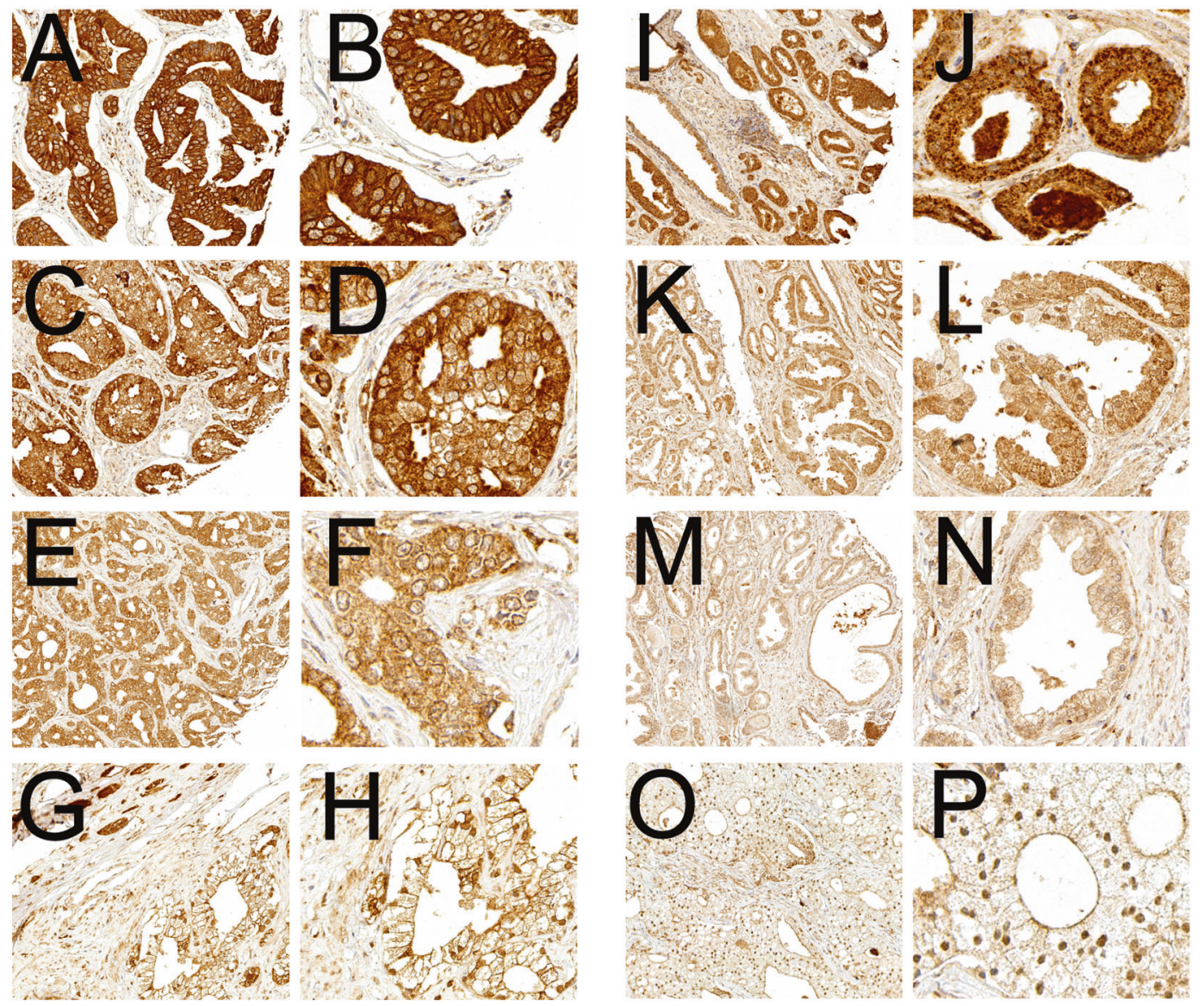

Fig. 1 SOAT1- and SOAT2-immunohistochemistry of high-risk prostate-cancer. High-risk prostate cancer tissue with strong $(\mathrm{H}$-score $3, \mathrm{~A}+$ $\mathrm{B}, \mathrm{I}+\mathrm{J})$, moderate $(\mathrm{H}$-score $2, \mathrm{C}+\mathrm{D}, \mathrm{K}+\mathrm{L})$, low $(\mathrm{H}$-score $1, \mathrm{E}+\mathrm{F}, \mathrm{M}+\mathrm{N})$ and negative $(\mathrm{H}$-score $0, \mathrm{G}+\mathrm{H}, \mathrm{O}+\mathrm{P})$ expression of SOAT1 $(\mathbf{A}-\mathrm{H})$ and SOAT2 (I-P).
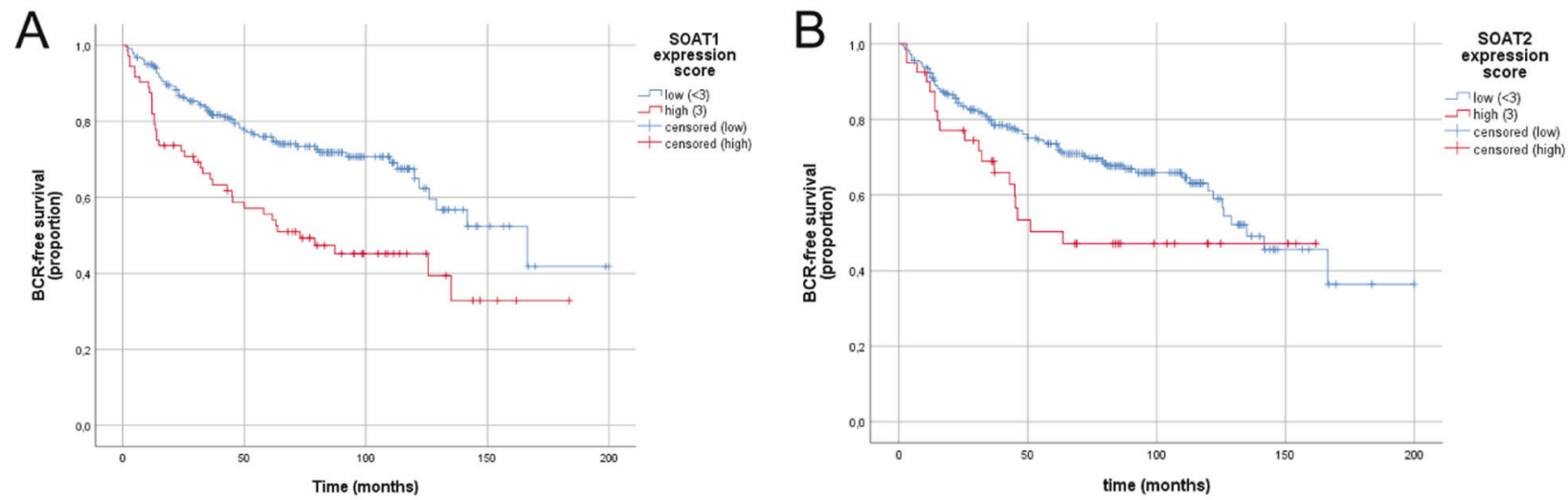

Fig. 2 Kaplan-Meier plots of SOAT1 and SOAT2 on BCR. Strong SOAT1 expression (H-score 3, A) is highly associated with significantly shorter BCR free survival compared to low SOAT1 expression (H-score $<3)$, Log-rank $p<0.001$. SOAT2 expression (B) is not significantly associated with BCR-free survival. BCR-free survival = biochemical recurrence free survival.

While clinical progression and survival are more robust parameters of prognosis, biochemical recurrence is a surrogate marker of more aggressive clinical course and has been used in similar studies due to the small proportion of patients who experience one of those end points. In addition, biochemical recurrence impacts strongly on further treatment which may mask a biologically meaningful role of a given marker. Thus microRNA221 has been identified as a prognostic marker and but is not ideally suited for routine clinical use.
Of note, high SOAT1 protein expression has already been demonstrated to correlate with a worse prognosis in hepatocellular carcinoma [36] and adrenocortical carcinoma [37].

Our data add high risk PCa to those malignancies where high SOAT1 expression is associated with earlier BCR. On the other hand, we did not observe association of SOAT1 expression with clinical recurrence and disease-specific death, most likely due to small percentages of patients who experienced clinical progression (14.4\%) or died of high risk PCa (7.2\%). Clinically, BCR triggers 
Table 2. Impact of SOAT1 and SOAT2 expression on survival.

\begin{tabular}{|c|c|c|c|c|c|c|c|c|c|}
\hline \multirow[b]{2}{*}{ Variable } & \multicolumn{3}{|c|}{ Univariate analysis } & \multicolumn{3}{|c|}{ Multivariate analysis SOAT1 } & \multicolumn{3}{|c|}{ Multivariate analysis SOAT2 } \\
\hline & $\begin{array}{l}\text { Mean survival } \\
\text { (months) }\end{array}$ & $95 \% \mathrm{Cl}$ & $\begin{array}{l}p(\text { Log- } \\
\text { rank) }\end{array}$ & $\mathrm{HR}$ & $95 \% \mathrm{Cl}$ & $\begin{array}{l}p \text { (Cox } \\
\text { regression) }\end{array}$ & $\mathrm{HR}$ & $95 \% \mathrm{Cl}$ & $\begin{array}{l}p \text { (Cox } \\
\text { regression) }\end{array}$ \\
\hline \multicolumn{10}{|l|}{ Age } \\
\hline$<66.0$ & 126 & $111-141$ & 0.931 & & & & & & \\
\hline$\geq 66.0$ & 112 & $100-124$ & & 1.10 & $0.60-1.37$ & 0.64 & 0.85 & $0.56-1.29$ & 0.43 \\
\hline \multicolumn{10}{|c|}{ Pre-operative PSA (Quartiles) } \\
\hline$\leq 21.38$ & 104 & $87-121$ & 0.054 & & & & & & \\
\hline $21.39-29.60$ & 154 & $136-172$ & & 0.53 & $0.28-1.00$ & 0.05 & 0.45 & $0.24-0.85$ & 0.01 \\
\hline $29.61-50.63$ & 124 & $103-145$ & & 0.88 & $0.51-1.54$ & 0.66 & 0.84 & $0.48-1.45$ & 0.52 \\
\hline$>50.63$ & 101 & 83-119 & & 1.24 & $0.73-2.10$ & 0.43 & 1.04 & $0.62-1.76$ & 0.88 \\
\hline \multicolumn{10}{|c|}{ Clinical stage } \\
\hline T2 (58) & 142 & $120-164$ & 0.135 & & & & & & \\
\hline T3(202) & 116 & $103-128$ & & 1.57 & $0.88-2.87$ & 0.13 & 1.32 & $075-2.34$ & 0.34 \\
\hline T4 (43) & 94 & $74-114$ & & 2.23 & $1.03-5.01$ & 0.042 & 1.81 & $0.87-3.78$ & 0.11 \\
\hline \multicolumn{10}{|c|}{ Gleason score } \\
\hline$\leq 7(221)$ & 131 & $118-144$ & 0.115 & & & & & & \\
\hline $8-10(63)$ & 91 & $76-106$ & & 1.57 & $0.99-2.49$ & 0.06 & 1.31 & $0.82-2.09$ & 0.27 \\
\hline \multicolumn{10}{|c|}{ Resection margins } \\
\hline Ro (121) & 139 & $123-154$ & 0.462 & & & & & & \\
\hline R1 (147) & 110 & $97-123$ & & 1.24 & $0.76-2.01$ & 0.40 & 1.22 & $0.72-1.97$ & 0.50 \\
\hline RX (35) & 104 & $86-122$ & & 1.03 & $0.51-2.06$ & 0.94 & 0.93 & $0.46-1.87$ & 0.83 \\
\hline \multicolumn{10}{|c|}{ Lymph node status } \\
\hline No (209) & 121 & $107-133$ & 0.190 & & & & & & \\
\hline N1 (94) & 126 & $109-143$ & & 0.80 & $0.50-1.31$ & 0.39 & 0.70 & $0.43-1.17$ & 0.17 \\
\hline \multicolumn{10}{|c|}{ SOAT1 expression score } \\
\hline$<3(218)$ & 134 & $120-148$ & & & & & & & \\
\hline $3(72)$ & 93 & 74-112 & $<0.001$ & 2.40 & $1.57-3.68$ & $<0.001$ & & & \\
\hline \multicolumn{10}{|c|}{ SOAT2 expression score } \\
\hline$<3(247)$ & 125 & $113-139$ & & & & & & & \\
\hline $3(40)$ & 91 & $88-136$ & 0.05 & & & & 1.57 & $0.93-2.64$ & 0.09 \\
\hline Year of surs & & & & 1.00 & $0.93-1.08$ & 0.967 & 0.97 & $0.90-1.05$ & 0.44 \\
\hline
\end{tabular}

Univariate survival analysis using Log-Rank Test and multivariate Cox-regression analysis showed a significant impact of strong SOAT1 expression score on biochemical recurrence free survival.

Statistically significant associations are highlighted in bold.

additional treatment that is likely to impact on clinical recurrence and survival.

SOAT1 catalyzes the esterification of free cholesterol to $C E$, which is subsequently stored in LDs. Free cholesterol accumulation is toxic e.g., through the induction of endoplasmic reticulum stress and hence cholesterol esterification favors cell survival. Abundancy of cholesterol has been shown to increase during PCa progression [38] and CE are enriched in LDs of human PCa tissues but not in normal prostates or benign prostatic hyperplasia [6]. Experimental inhibition of SOAT1 and consequently the esterification of free cholesterol reduced cell viability in a PCa cell culture model. Tumor growth [6] and metastasis was reduced in an orthotopic mouse model [7].

Our study now translates these experimental findings into a clinical setting and has the strength of a clinically well defined high risk $\mathrm{PCa}$ cohort which requires treatment and hence is not confounded by low-risk cases with a more benign course due to a different underlying tumoral biology. Although the two cohorts analyzed here were acquired and processed in two different centers and differ significantly in terms of variables associated with prognosis (Table S1), the high prognostic value of SOAT1 expression in the combined cohort strengthens its role of SOAT1 expression as factor of prognosis.

Data published by Stopsack et al. seemingly contradict our findings. Rather unexpectedly, they found low SOAT1 and low LDLR but high SQLE mRNA expression in clinical cohort studies to be associated with a higher Gleason score. Hence high mRNA expression of these genes was concluded to be associated with increased PCa dedifferentiation [39]. First, this study has the significant shortcoming that only mRNA expression was assessed which does not automatically correlate with protein expression and may largely be affected by stroma components [40, 41]. Second, decreased SOAT1 gene methylation in PCa has been demonstrated which results in increased mRNA and protein expression in PCa [42]. Third, aberrant lipid metabolism is well established as a factor for PCa aggressiveness [43] and it would be surprising if low instead of high expression of the enzymes involved would be correlated with increased PCa dedifferentiation. The positive correlation of SOAT1 with enzymes involved in lipid metabolism (Fig. S1, Table S2) confirms our finding of high SOAT1 expression as a factor of poorer prognosis in high risk PCa as demonstrated for SQLE in another study [44]. In addition, our data suggest that SOAT1-dependent 
cholesterol metabolism and AR signaling may be functionally related and contribute to PCa progression. Importantly, increased intratumoral androgen synthesis from cholesterol associated with higher expression of genes involved in cholesterol metabolism have been shown [45] and potentially contribute to the development of castration refractory tumors.

The main limitation of our study is the retrospective study design and the limitation of samples to only two centers. Potential confounders such as statin use [46] but also follow-up treatment were unavailable which is expected to have varied over the relatively long recruitment period. Nevertheless, we consider the combined study cohort with its large sample number representative of the spectrum of high risk PCa. Importantly, very recently similar observations as ours have confirmed our findings [47].

While in the past, SOAT inhibition has been explored for the treatment of atherosclerosis [48], more recently, the SOAT1 inhibitor nevanimibe was tested in a phase I clinical trial as a treatment of adrenocortical carcinoma (ACC). Although the further development in ACC was discontinued due to the absence of clinically meaningful antitumoral activity, little dose-limitingtoxicities occurred and the drug was rather well tolerated [17]. Of note, SOAT1 expression was not reported in the study cohort although SOAT1 expression was shown to be very heterogenous in adrenocortical carcinoma [14, 37, 49]. Since in an exploratory experiment nevanimibe treatment of LNCaP (Fig. S3A) and PC3 PCa cells (Fig. S3B) significantly decreased viability, a clinical trial of the available SOAT1 inhibitor nevanimibe might present a possible treatment option in high risk $\mathrm{PCa}$ patients with high SOAT1 expression and biochemical progression.

\section{CONCLUSION}

Our results clearly show a high prognostic value of SOAT1 protein expression in high risk PCa patients which helps to identify a subgroup of high risk PCa patients rather likely to experience BCR. SOAT1 expression may be associated with increased cholesterol metabolism and potentially androgen signaling in PCa. Patients with high SOAT1 expression might be good candidates for pharmacological SOAT1 inhibition. SOAT1 inhibitors have been clinically developed and may be repurposed for PCa treatment.

\section{REFERENCES}

1. Culp MB, Soerjomataram I, Efstathiou JA, Bray F, Jemal A. Recent global patterns in prostate cancer incidence and mortality rates. Eur Urol. 2020;77:38-52.

2. Ito K, Oki R, Sekine Y, Arai S, Miyazawa Y, Shibata Y, et al. Screening for prostate cancer: History, evidence, controversies and future perspectives toward individualized screening. Int J Urol: Off J Jpn Urological Assoc. 2019;26:956-70.

3. Fitzmaurice C, Allen C, Barber RM, Barregard L, Bhutta ZA, Brenner H, et al. Global, regional, and national cancer incidence, mortality, years of life lost, years lived with disability, and disability-adjusted life-years for 32 cancer groups, 1990 to 2015: a systematic analysis for the global burden of disease study. JAMA Oncol. 2017;3:524-48.

4. Tosco L, Laenen A, Briganti A, Gontero P, Karnes RJ, Bastian PJ, et al. The EMPaCT classifier: a validated tool to predict postoperative prostate cancer-related death using competing-risk analysis. Eur Urol Focus. 2018;4:369-75.

5. Pavlova NN, Thompson CB. The emerging hallmarks of cancer metabolism. Cell Metab. 2016;23:27-47.

6. Yue S, Li J, Lee SY, Lee HJ, Shao T, Song B, et al. Cholesteryl ester accumulation induced by PTEN loss and PI3K/AKT activation underlies human prostate cancer aggressiveness. Cell Metab. 2014;19:393-406.

7. Lee HJ, Li J, Vickman RE, Li J, Liu R, Durkes AC, et al. Cholesterol esterification inhibition suppresses prostate cancer metastasis by impairing the $\mathrm{Wnt} / \mathrm{\beta}$-catenin pathway. Mol Cancer Res. 2018;16:974-85.

8. Locke JA, Wasan KM, Nelson CC, Guns ES, Leon CG. Androgen-mediated cholesterol metabolism in $\mathrm{LNCaP}$ and $\mathrm{PC}-3$ cell lines is regulated through two different isoforms of acyl-coenzyme A:Cholesterol Acyltransferase (ACAT). Prostate. 2008;68:20-33.

9. Leon CG, Locke JA, Adomat HH, Etinger SL, Twiddy AL, Neumann RD, et al. Alterations in cholesterol regulation contribute to the production of intratumoral androgens during progression to castration-resistant prostate cancer in a mouse xenograft model. Prostate. 2010;70:390-400.

10. Seo T, Oelkers PM, Giattina MR, Worgall TS, Sturley SL, Deckelbaum RJ. Differential modulation of ACAT1 and ACAT2 transcription and activity by long chain free fatty acids in cultured cells. Biochemistry. 2001;40:4756-62.

11. Beloribi-Djefaflia S, Vasseur S, Guillaumond F. Lipid metabolic reprogramming in cancer cells. Oncogenesis. 2016;5:e189.

12. Temel RE, Hou L, Rudel LL, Shelness GS. ACAT2 stimulates cholesteryl ester secretion in apoB-containing lipoproteins. J lipid Res. 2007;48:1618-27.

13. Parini $P$, Davis $M$, Lada AT, Erickson SK, Wright TL, Gustafsson U, et al. ACAT2 is localized to hepatocytes and is the major cholesterol-esterifying enzyme in human liver. Circulation. 2004;110:2017-23.

14. Sbiera S, Leich E, Liebisch G, Sbiera I, Schirbel A, Wiemer L, et al. Mitotane inhibits sterol-O-Acyl transferase 1 triggering lipid-mediated endoplasmic reticulum stress and apoptosis in adrenocortical carcinoma cells. Endocrinology. 2015;156:3895-908.

15. LaPensee CR, Mann JE, Rainey WE, Crudo V, Hunt SW 3rd, Hammer GD. ATR-101, a selective and potent inhibitor of Acyl-CoA acyltransferase 1, induces apoptosis in $\mathrm{H} 295 \mathrm{R}$ adrenocortical cells and in the adrenal cortex of dogs. Endocrinology. 2016;157:1775-88.

16. Langlois DK, Fritz MC, Schall WD, Bari Olivier N, Smedley RC, Pearson PG, Bailie MB, Hunt SW 3rd. ATR-101, a selective ACAT1 inhibitor, decreases ACTHstimulated cortisol concentrations in dogs with naturally occurring Cushing's syndrome. BMC Endocr Disord. 2018;18:24.

17. Smith DC, Kroiss M, Kebebew E, Habra MA, Chugh R, Schneider BJ, et al. A phase 1 study of nevanimibe $\mathrm{HCl}$, a novel adrenal-specific sterol O-acyltransferase 1 (SOAT1) inhibitor, in adrenocortical carcinoma. Investigational N. Drugs. 2020:38:1421-9.

18. Briganti A, Karnes RJ, Gandaglia G, Spahn M, Gontero P, Tosco L, et al. Natural history of surgically treated high-risk prostate cancer. Urologic Oncol. 2015;33:163.e167-113.

19. Kneitz B, Krebs M, Kalogirou C, Schubert M, Joniau S, van Poppel H, et al. Survival in patients with high-risk prostate cancer is predicted by miR-221, which regulates proliferation, apoptosis, and invasion of prostate cancer cells by inhibiting IRF2 and SOCS3. Cancer Res. 2014;74:2591-603.

20. Abida W, Cyrta J, Heller G, Prandi D, Armenia J, Coleman I, et al. Genomic correlates of clinical outcome in advanced prostate cancer. Proc Natl Acad Sci. 2019;116:11428-36

21. Gao J, Aksoy BA, Dogrusoz U, Dresdner G, Gross B, Sumer SO, et al. Integrative analysis of complex cancer genomics and clinical profiles using the cBioPortal. Sci Signal. 2013;6:pl1.

22. Sbiera S, Schmull S, Assie G, Voelker HU, Kraus L, Beyer M, et al. High diagnostic and prognostic value of steroidogenic factor-1 expression in adrenal tumors. Clin Endocrinol Metab. 2010;95:E161-71.

23. Hieronymus H, Lamb J, Ross KN, Peng XP, Clement C, Rodina A, et al. Gene expression signature-based chemical genomic prediction identifies a novel class of HSP90 pathway modulators. Cancer Cell. 2006;10:321-30.

24. National Library of Medicine (US), National Center for Biotechnology Information; 2004-. PubChem Pathway Summary for Pathway R-HSA-191273, Cholesterol biosynthesis, Source: Reactome; [cited 2021 Apr. 9). Available from: http:// pubchem.ncbi.nlm.nih.gov/pathway/Reactome:R-HSA-191273.

25. Cerami et al. The cBio Cancer Genomics Portal: An Open Platform for Exploring Multidimensional Cancer Genomics Data. Cancer Discovery. 2012;2:401.

26. Chaturvedi AP, Dehm SM. Androgen receptor dependence. Adv Exp Med Biol. 2019;1210:333-50.

27. Cooperberg MR, Cowan J, Broering JM, Carroll PR. High-risk prostate cancer in the United States, 1990-2007. World J Urol. 2008;26:211-8.

28. Cooperberg MR, Broering JM, Carroll PR. Time trends and local variation in primary treatment of localized prostate cancer. J Clin Oncol: Off J Am Soc Clin Oncol. 2010;28:1117-23.

29. Terakawa T, Katsuta E, Yan L, Turaga N, McDonald KA, Fujisawa M, et al. High expression of SLCO2B1 is associated with prostate cancer recurrence after radical prostatectomy. Oncotarget. 2018;9:14207-18.

30. Bastian PJ, Boorjian SA, Bossi A, Briganti A, Heidenreich A, Freedland SJ, et al. High-risk prostate cancer: from definition to contemporary management. Eur Urol. 2012;61:1096-106.

31. Joniau S, Van der Eeckt K, Briganti A, Gontero P, Van Bruwaene S, Jeffrey, et al. Current role of surgery for high risk prostate cancer. Archivos espanoles de urologia. 2013;66:259-73. 259-274

32. Chang AJ, Autio KA, Roach M, Scher HI. "High-risk" prostate cancer: classification and therapy. Nat Rev Clin Oncol. 2014;11:308-23.

33. Pound CR, Partin AW, Eisenberger MA, Chan DW, Pearson JD, Walsh PC. Natural history of progression after PSA elevation following radical prostatectomy. Jama. 1999;281:1591-7. 
34. Freedland SJ, Humphreys EB, Mangold LA, Eisenberger M, Dorey FJ, Walsh PC, et al. Risk of prostate cancer-specific mortality following biochemical recurrence after radical prostatectomy. Jama. 2005;294:433-9.

35. Krebs M, Behrmann C, Kalogirou C, Sokolakis I, Kneitz S, Kruithof-de Julio M et al. M. et al. miR-221 augments TRAIL-mediated apoptosis in prostate cancer cells by inducing endogenous TRAIL expression and targeting the functional repressors SOCS3 and PIK3R1. BioMed Res Int. 2019;2019:6392748

36. Jiang $Y$, Sun $A$, Zhao $Y$, Ying $W$, Sun $H$, Yang $X$, et al. Proteomics identifies new therapeutic targets of early-stage hepatocellular carcinoma. Nature. 2019;567:257-61.

37. Lacombe AMF, Soares IC, Mariani BMP, Nishi MY, Bezerra-Neto JE, Charchar HDS, et al. Sterol O-Acyl Transferase 1 as a Prognostic Marker of Adrenocortical Carcinoma. Cancers 2020; 12.

38. Krycer JR, Brown AJ. Cholesterol accumulation in prostate cancer: a classic observation from a modern perspective. Biochimica et biophysica acta. 2013;1835:219-29.

39. Stopsack KH, Gerke TA, Andrén O, Andersson SO, Giovannucci EL, Mucci LA, et al. Cholesterol uptake and regulation in high-grade and lethal prostate cancers. Carcinogenesis. 2017;38:806-11.

40. Gygi SP, Rochon Y, Franza BR, Aebersold R. Correlation between protein and mRNA abundance in yeast. Mol Cell Biol. 1999;19:1720-30.

41. Maier T, Güell M, Serrano L. Correlation of mRNA and protein in complex biological samples. FEBS Lett. 2009;583:3966-73.

42. Latonen L, Afyounian E, Jylhä A, Nättinen J, Aapola U, Annala M, et al. Integrative proteomics in prostate cancer uncovers robustness against genomic and transcriptomic aberrations during disease progression. Nat Commun. 2018;9:1176.

43. Galbraith L, Leung HY, Ahmad I. Lipid pathway deregulation in advanced prostate cancer. Pharm Res. 2018;131:177-84.

44. Stopsack KH, Gerke TA, Sinnott JA, Penney KL, Tyekucheva S, Sesso HD, et al. Cholesterol metabolism and prostate cancer lethality. Cancer Res. 2016;76:4785-90.

45. Dillard PR, Lin MF, Khan SA. Androgen-independent prostate cancer cells acquire the complete steroidogenic potential of synthesizing testosterone from cholesterol. Mol Cell Endocrinol. 2008;295:115-20.

46. Raittinen P, Niemisto K, Pennanen E, Syvala H, Auriola S, Riikonen J, et al. Circulatory and prostatic tissue lipidomic profiles shifts after high-dose atorvastatin use in men with prostate cancer. Sci Rep. 2020;10:12016.

47. Liu Y, Wang Y, Hao S, Qin Y, Wu Y. Knockdown of sterol O-acyltransferase 1 (SOAT1) suppresses SCD1-mediated lipogenesis and cancer procession in prostate cancer. Prostaglandins Other Lipid Mediators. 2021;153:106537.

48. Nissen SE, Tuzcu EM, Brewer HB, Sipahi I, Nicholls SJ, Ganz P, et al. Effect of ACAT inhibition on the progression of coronary atherosclerosis. N. Engl J Med. 2006;354:1253-63.

49. Weigand I, Altieri B, Lacombe A, Basile V, Kircher S, Landwehr L-S et al. Expression of SOAT1 in adrenocortical carcinoma and response to mitotane monotherapy: an ENSAT multicenter study. J Clin Endocrinol Metabol. 2020; 105:2642-53.

\section{ACKNOWLEDGEMENTS}

The results shown here are in part based upon data generated by the TCGA Research. Network: https://www.cancer.gov/tcga. We are grateful to Sabine Kendl for expert technical assistance.

\section{AUTHOR CONTRIBUTIONS}

M.Kro., M.S. and M.Kre. drafted the study, M.S., S.J. and B.K provided tissue specimens and clinical data, C.E., S.S., I.S. and M.Kre. performed experiments, C.E., M.Kro. and I.W. analysed data, C.E., I.W., M.Kre and M.Kro. wrote the manuscript, and M.Kro. supervised the study. All authors contributed to the intellectual content of the work and critically revised and approved the manuscript.

\section{FUNDING}

This work has been supported by grants from the DFG German Research Foundation (project numbers 237292849 and 314061271 to M.F. and M.Kro and 269025972 to B.K.) and the Else Kröner-Fresenius-Stiftung (grant 2016 A96 to S.S. and M.Kro. and a fellowship to M. Kre. in the Else Kröner Integrative Clinician Scientist College for Translational Immunology, University Hospital Wuerzburg). Open Access funding enabled and organized by Projekt DEAL.

\section{COMPETING INTERESTS}

The authors declare no competing interests.

\section{ADDITIONAL INFORMATION}

Supplementary information The online version contains supplementary material available at https://doi.org/10.1038/s41391-021-00431-3.

Correspondence and requests for materials should be addressed to M.K.

Reprints and permission information is available at http://www.nature.com/ reprints

Publisher's note Springer Nature remains neutral with regard to jurisdictional claims in published maps and institutional affiliations.

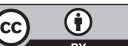

Open Access This article is licensed under a Creative Commons Attribution 4.0 International License, which permits use, sharing, adaptation, distribution and reproduction in any medium or format, as long as you give appropriate credit to the original author(s) and the source, provide a link to the Creative Commons license, and indicate if changes were made. The images or other third party material in this article are included in the article's Creative Commons license, unless indicated otherwise in a credit line to the material. If material is not included in the article's Creative Commons license and your intended use is not permitted by statutory regulation or exceeds the permitted use, you will need to obtain permission directly from the copyright holder. To view a copy of this license, visit http://creativecommons. org/licenses/by/4.0/.

(c) The Author(s) 2021 